\title{
Highlights of recent advances in functional coordination polymers made in China
}

\author{
DU Miao ${ }^{1}$, CHEN YongQiang ${ }^{2} \&$ BU XianHe ${ }^{2 *}$ \\ ${ }^{1}$ College of Chemistry, Tianjin Key Laboratory of Structure and Performance for Functional Molecule, Tianjin Normal University, Tianjin \\ 300387, China: \\ ${ }^{2}$ Department of Chemistry, Tianjin Key Laboratory of Metal- and Molecule-Based Material Chemistry, Nankai University, Tianjin 300071, China
}

Received March 29, 2011; accepted April 6, 2011

Coordination polymers (or metal-organic frameworks, MOFs), as one of the essences of crystal engineering and coordination chemistry, have attracted considerable attention from chemists, physicists, and materials scientists for the development of hybrid crystalline solids with novel supramolecular structures and physicochemical properties. In particular, research on functional coordination polymers has evolved considerably during the last decade [1]. In this context, Science China Chemistry and Chinese Science Bulletin, as internationally recognized and influential academic journals, have provided a stage for chemists in China to demonstrate their significant contributions to this field. Current research is mostly concentrated on the potential applications of such network-based complexes as magnetic, fluorescent, adsorption, optoelectronic, drug delivery, and multifunctional molecule-based materials.

One of the flourishing themes in coordination polymers is the design and synthesis of diversiform molecule-based magnetic materials with interesting structures and properties [2]. In this regard, Gao et al. [3] have successfully prepared two three-dimensional (3D) $\mathrm{Mn}^{\mathrm{II}}-\mathrm{W}^{\mathrm{IV}}$ and $\mathrm{Cu}^{\mathrm{II}}-\mathrm{W}^{\mathrm{IV}}$ coordination systems based on octacyanotungstate (IV) and 4,4'-bipyridine dioxide. Antiferromagnetic coupling is observed between the $\mathrm{Mn}^{\mathrm{II}}$ ions, while a weak ferromagnetic interaction exists between the $\mathrm{Cu}^{\mathrm{II}}$ centers. In a similar vein, Song and co-workers synthesized two bimetallic $\mathrm{Mn}^{\mathrm{II}}-\mathrm{W}^{\mathrm{IV}}$ coordination polymers with $1 \mathrm{D}$ chain and 3D open network structures [4]. Both complexes show weak antiferromagnetic interactions between adjacent $\mathrm{Mn}^{\mathrm{II}}$ ions because of the presence of long diamagnetic bridges. In addition, a novel heterospin $1 \mathrm{D}$ polymeric chain generated from $\mathrm{Cu}^{\mathrm{II}}$ and nitroxide radicals has been reported by Liao's group [5].

*Corresponding author (email: buxh@nankai.edu.cn)
This complex displays a ferromagnetic interaction between $\mathrm{Cu}^{\mathrm{II}}$ and nitroxide radicals, as well as a weak antiferromagnetic interaction between the $\mathrm{Cu}^{\mathrm{II}}$ centers.

Carboxylate-based polymeric coordination complexes represent an important class of magnetic materials. For example, $\mathrm{Bu}$ and co-workers [6] synthesized two isomorphous $3 \mathrm{D} \mathrm{Mn}{ }^{\mathrm{II}}$ and $\mathrm{Co}^{\mathrm{II}}$ coordination polymers using a mixture of benzoimidazol-1-yl-acetate and formate ligands. Both complexes display a new $(3,6)$-connected topological network and distinct magnetic behaviors, that is, spin-canted antiferromagnetism for the $\mathrm{Mn}^{\mathrm{II}}$ complex and antiferromagnetic coupling for the homologous $\mathrm{Co}^{\mathrm{II}}$ species, which are essentially based on the interactions between the metal ions and bridging carboxylate groups. Feng and co-workers [7] prepared a $2 \mathrm{D} \mathrm{Mn}^{\mathrm{II}}$ coordination polymer hydrothermally using 1,4-benzenebis(thioacetic acid) as bridging tectons and 1,10-phenanthroline as a chelating co-ligand. The layered structure contains linear trinuclear $\mathrm{Mn}^{\mathrm{II}}$ subunits that display weak antiferromagnetic interactions. Zuo and coworkers [8] prepared a $1 \mathrm{D}$ chain-like $\mathrm{Co}^{\mathrm{II}}$ coordination polymer with tetrathiafulvalene carboxylate that exhibits ferromagnetic interactions at low temperature between intrachain $\mathrm{Co}^{\mathrm{II}}$ ions bridged by carboxylate groups. The presence of bulky crown-ether segments and weak intermolecular $S \cdots S$ interactions causes this complex to have low electric conductivity. A $3 \mathrm{D}$ porous $\mathrm{Cu}^{\mathrm{II}} \mathrm{MOF}$ containing tris(2carboxyethyl)isocyanurate and piperazine was prepared by Hou and co-workers under hydrothermal conditions [9]. In the resulting $(3,8)$-connected network, the planar tetranuclear $\mathrm{Cu}^{\mathrm{II}}$ clusters formed via bridging hydroxyl and carboxylate anions act as 8-connected nodes and exhibit antiferromagnetic interactions. Using the connectivity of both carboxylate and triazole groups in 1H-1,2,3-triazole-4,5dicarboxylic acid, Su and co-workers [10] constructed a 3D 
$(3,4)$-connected $\mathrm{Co}^{\mathrm{II}}$ coordination polymer that displays antiferromagnetic coupling between the $\mathrm{Co}^{\mathrm{II}}$ ions. More interestingly, Zheng et al. [11] synthesized a $1 \mathrm{D} \mathrm{Co} \mathrm{Co}^{\mathrm{II}}$ helical complex with 6-phosphononicotinic acid, in which the metal centers bridged by phosphonate groups show antiferromagnetic interactions. After excluding coordinated and lattice water, visible changes are observed in the color and structure of the dehydrated material, which shows the metamagnetic behavior at low temperature. Coordination polymers containing metal ions other than those of transition metals have also been prepared. Zhao and co-workers [12] produced a $3 \mathrm{D} \mathrm{Gd}^{\mathrm{III}}$ coordination framework with $1 \mathrm{D}$ channels, in which ferromagnetic coupling exists between adjacent metal centers bridged by the carboxylate groups of 2,6-naphthalenedicarboxylate ligands. Two porous $\mathrm{Ce}^{\mathrm{III}}$ MOFs containing two different carboxylic acid derivatives have been reported by Wang et al. [13]. The magnetic properties of both pillared-layer coordination frameworks were also studied.

On the other hand, fluorescent MOFs are of great significance because of their high thermal stability and favorable metal-ligand interactions for structural and functional regulation. As a representative type of photoluminescent material, coordination polymers containing $\mathrm{d}^{10}$ metal ions have received particular consideration. For example, Sun and co-workers have reported two novel $\mathrm{Zn}^{\mathrm{II}}$ and $\mathrm{Cd}^{\mathrm{II}}$ coordination polymers containing 4-imidazole-benzoate, which display a 2-fold interpenetrating $2 \mathrm{D}(4,4)$ layered structure that crystallizes in the chiral group $P 4_{1} 22$, and a 4-fold interpenetrating 3D porous framework with diamond topology, respectively. The acentric $\mathrm{Zn}^{\mathrm{II}}$ coordination polymer shows modest powder second-harmonic-generation (SHG) activity, making it a good candidate for non-linear optical applications. With regard to their photoluminescence properties, only the $\mathrm{Cd}^{\mathrm{II}}$ species exhibits enhanced ligand-based fluorescent emission at $480 \mathrm{~nm}$ [14]. Cheng and co-workers [15] prepared two $1 \mathrm{D}$ zigzag $\mathrm{Zn}^{\text {II }}$ coordination polymers and one 2D grid-like layered $\mathrm{Cd}^{\mathrm{II}}$ complex using 2,5-dihydroxy-pbenzenediacetic acid and different $\mathrm{N}, \mathrm{N}^{\prime}$-chelating co-ligands. These polymeric materials exhibit solid state fluorescent emissions that originate from charge transfer between the metal centers and organic ligands. Likewise, Wang and coworkers prepared two $2 \mathrm{D} \mathrm{Zn}^{\mathrm{II}}$ coordination polymers with different helical subunits as well as one 1D helical coordination array using 5,5'-dithiobis(2-nitrobenzoic acid) and secondary $\mathrm{N}$-donor bridging or chelating co-ligands. All the complexes exhibit ligand-based solid state fluorescent emission where the metal ions enhance the intensity of emission [16]. Chen et al. [17] also demonstrated that rigid dicarboxylate building blocks could facilitate fluorescent enhancement, by exploring the luminescent properties of three $\mathrm{Cd}^{\mathrm{II}}$ coordination polymers containing different rigid/flexible dicarboxylate species and $\mathrm{N}$-donor chelating co-ligands. The two 2D layered $\mathrm{Cd}^{\mathrm{II}}$ coordination polymers based on 2-(2-pyridinyl-benzimidazolyl)acetic acid and 5-hydroxy-isophthalic acid as a co-ligand prepared by Zheng and co-workers [18] exhibit red shifts of fluorescence maxima compared with that of the free ligand. Su's research group [19] obtained a 3D MOF from the hydrothermal reaction of $\mathrm{Zn}{ }^{\mathrm{II}}$ with 5-aminotetrazole that shows a diamond network topology, hexagonal channels, and strong blue fluorescence at room temperature. In addition, Lang and co-workers [20] prepared four different 1D and 2D $\left[\mathrm{Cu}_{n} \mathrm{I}_{n}\right]$-based coordination polymers using flexible benzimidazolyl derivatives. These polymeric complexes exhibit strong solid state emissions at 507-597 nm with different origins, so may be used as green-emitting luminescent materials. As well as $\mathrm{d}^{10}$ coordination systems, lanthanideorganic frameworks can also display attractive photoluminescence behavior. For example, Zhao et al. [12] reported a $2 \mathrm{D} \mathrm{Eu}{ }^{\mathrm{III}}$ coordination polymer that exhibits the characteristic red emission of $\mathrm{Eu}^{\mathrm{III}}$ in DMF solution.

The applications of coordination polymers have recently been extended to areas such as gas adsorption, drug carriers, and semiconductors. In this context, a novel $2 \mathrm{D}$ layered $\mathrm{Ni}^{\mathrm{II}}$ organo-diphosphonate containing 4,4'-bipyridine co-ligand with the capability to adsorb alkaline gas was recently produced by hydrothermal synthesis [21]. Sun and co-workers reported four 2D and 3D bismuth citrate coordination polymers based on dimeric building units, with the inclusion of protonated ethylenediamine and pyridine guest moieties [22]. A possible pharmacokinetic pathway for bismuth citrate-based drugs was proposed, in which release of encapsulated drugs from the porous matrix of bismuth citrate may occur at low $\mathrm{pH}$ value. As a result, these polymeric systems can be considered as a new type of hybrid material with potential application in drug carrier. Niu and co-workers [23] prepared two 1D $\mathrm{Fe}(\mathrm{II} / \mathrm{III})$ coordination polymers with mixed ligands. These complexes show $p$-type semiconductor characteristics, as confirmed by their surface photovoltage spectra exhibiting positive surface photovoltaic responses in the range of 300-600 nm. These results may aid in developing novel photoelectric materials.

Very recently, the rational design and preparation of coordination polymers with multiple functions has received increasing research interest [1]. With this in mind, Qiu's group [24] prepared a 3D Mn ${ }^{\mathrm{II}}$ coordination framework with rutile-type network topology using 8-hydroxylquinoline-5sulfonate. This framework exhibits strong antiferromagnetic interactions between adjacent $\mathrm{Mn}^{\mathrm{II}}$ centers and also fluoresces intensely at $568 \mathrm{~nm}$ because of ligand-to-metal charge transfer. Chen and co-workers [25] synthesized a 3D rutile-type $\mathrm{Zn}^{\mathrm{II}}$ coordination polymer with 1,3,5-benzenetricarboxylate, in which DMF solvent molecules and $\mathrm{Me}_{2} \mathrm{NH}_{2}{ }^{+}$cations are accommodated. After removing the $\mathrm{DMF}$ molecules by heating at $160^{\circ} \mathrm{C}$, a structural transformation occurs to afford a new phase that exhibits proton conductivity. Further heat treatment at $260^{\circ} \mathrm{C}$ induces the release of counter cations in their neutral form through a proton transfer process. The resulting porous solid shows a 
moderate uptake of $\mathrm{CO}_{2}$ gas, and can adsorb methanol and exclude acetone molecules. The same group also prepared a $(3,12)$-connected porous framework with octacobalt clusters from $\mathrm{CoSO}_{4}$ and 2,6-di-p-carboxyphenyl-4,4'-bipyridine. This framework displays spin-glassy behavior as well as gas adsorption properties toward $\mathrm{N}_{2}$ and $\mathrm{H}_{2}$ after activation by heating [26]. Additionally, Cao and co-workers [27] synthesized a $3 \mathrm{D}$ porous coordination framework from $\mathrm{Zn}^{\mathrm{II}}$ and tetrakis[4-(carboxyphenyl)oxamethyl]methane acid with chiral building units, which can be regarded as a multifunctional crystalline material with ferroelectric and SHG activity as well as gas adsorption properties. Wang et al. [28] showed that metal-formate coordination frameworks may exhibit diverse topological networks depending on the size, shape, charge, and H-bonding geometries of the protonated amine templates. These molecule-based materials display promising magnetic, dielectric, porous, and optical properties.

The enormous attention devoted to coordination polymers originates from their diverse and adjustable network architectures as well as their associated properties and potential applications. The current research developments in this field, both at home and abroad, indicate a clear trend away from the initial random synthesis and structural characterization of coordination polymers to the design, prediction, and controlled preparation of desired functional systems. The latest progress has been production of advanced crystalline materials with multiple functions. Undoubtedly, the original results published in journals such as Science China Chemistry and Chinese Science Bulletin by Chinese chemists are of great significance and influence the overall direction of research in this field, where an abundance of developments remain to be made.

This work was supported by the National Natural Science Foundation of China (21031002).

1 Long J R, Yaghi O M. The pervasive chemistry of metal-organic frameworks. Chem Soc Rev, 2009, 38: 1213-1214

2 Huang Y G, Jiang F L, Hong M C. Magnetic lanthanide-transitionmetal organic-inorganic hybrid materials: From discrete clusters to extended frameworks. Coord Chem Rev, 2009, 253: 2814-2834

3 Yuan M, Gao S, Zhao F, et al. Synthesis, crystal structures, and magnetic properties of two three-dimensional octacyanotungstate(IV)-based bimetallic frameworks with 4,4'-bipyridine dioxide (4,4'-dpdo). Sci China Ser B-Chem, 2009, 52: 266-275

4 Liu B L, Xiao H P, Song Y, et al. Two bimetallic W(IV)-Mn(II) complexes based on octacyanometallates: structures and magnetic properties. Sci China Ser B-Chem, 2009, 52: 1801-1807

5 Ma Y, Chen X P, Cao D, et al. Structure and magnetic properties of a novel heterospin 1-D chain complex containing both $\mathrm{Cu}(\mathrm{II})$ and nitroxide radicals as spin branches. Sci China Ser B-Chem, 2009, 52: $1438-1443$

6 Hu B W, Zhao J P, Yang Q, et al. Synthesis, structure and magnetic properties of two new coordination polymers with carboxylate-substituted benzoimidazole ligands. Sci China Ser B-Chem, 2009, 52: 1451-1455

7 Wang X J, Yin J L, Chen J, et al. A new 2D layer manganese(II) complex assembled by flexible 1,4-benzenebis(thioacetic acid) ligand:
Synthesis, crystal structure and magnetic property. Chin J Inorg Chem, 2011, 27: 367-371

8 Chen Y, Li C H, Wang C F, et al. Synthesis, structure and physical properties of the one-dimensional chain complex of tetrathiafulvalene carboxylate. Sci China Ser B-Chem, 2009, 52: 1596-1601

9 Li H J, Zhang E P, Guo Q Q, et al. Structure and magnetism of a porous three-dimensional metal-organic framework based on planar tetranuclear copper(II) cluster units. Sci China Ser B-Chem, 2010, 53: 2118-2122

10 Yuan G, Shao K Z, Du D Y, et al. Synthesis and characterization of two novel coordination polymers based on the rigid $\mathrm{H}-1,2,3$-triazole-4,5dicarboxylic acid ligand. Sci China Ser B-Chem, 2010, 53: 2177-2182

11 Wang P F, Duan Y, Zheng L M. One-dimensional metal phosphonates based on 6-phosphononicotinic acid: A structural and magnetic study. Sci China Ser B-Chem, 2010, 53: 2112-2117

12 Li X H, Fang M, Zhao B. Synthesis, structure, and magnetic properties of two novel lanthanide-organic frameworks. Sci China Ser B-Chem, 2009, 52: 1456-1462

13 Zhu W H, Wang Z M, Gao S. Synthesis, structures and magnetism of two new 3D pillared-layer cerium mixed carboxylate porous metalorganic frameworks. Chem J Chin Univ, 2011, 32: 532-537

14 Bai Z S, Chen S S, Zhang Z H, et al. Two novel interpenetrated zinc(II) and cadmium(II) coordination polymers based on 4-imidazole-benzoate: Syntheses, crystal structures and properties. Sci China Ser B-Chem, 2009, 52: 459-464

15 Zhao L M, Ren P, Zhang Z J, et al. Synthesis, crystal structures and fluorescence properties of four mixed-ligands $\mathrm{Zn}$ (II) and $\mathrm{Cd}(\mathrm{II})$ coordination compounds. Sci China Ser B-Chem, 2009, 52: 1479-1484

16 Dong Z, Zhang W H, Wang Y Y, et al. Three new zinc(II) coordination polymers: Modulation of extended structures driven by assistant ligands. Chinese Sci Bull, 2009, 54: 4285-4290

17 Yue F, Jian M F, Chen X M. Structures and luminescence of three $\mathrm{Cd}(\mathrm{II})$ dicarboxylate coordination polymers. Chinese J Inorg Chem, 2009, 25: 1590-1594

18 Li J, Ji C C, Wang Z W, et al. Synthesis, crystal structure and fluorescence properties of $\mathrm{Cd}(\mathrm{II})$ compounds. Chinese J Inorg Chem, 2009, 25: 2083-2089

19 Qin J S, Lan Y Q, Wang H N, et al. Synthesis, characterization and luminescence of a new diamondoid MOF based on tetrazole derivative. Chem J Chin Univ, 2011, 32: 683-687

20 Li L L, Ren Z G, Lü X, et al. Construction of $\left[\mathrm{Cu}_{n} \mathrm{I}_{n}\right]$-based coordination polymers via flexible benzimidazolyl-based ligands. Sci China Ser B-Chem, 2010, 53: 2083-2090

21 Li H, Ren H, Zhang W W, et al. Synthesis, structure and gas adsorption property of a novel 2D layer nickel organo-diphosphonate compound. Chem J Chin Univ, 2011, 32: 28-31

22 Yang N, An Y, Cai J W, et al. Polymeric architectures of bismuth citrate based on dimeric building blocks. Sci China Ser B-Chem, 2010, 53: 2152-2158

23 Shi Z F, Jin J, Zhao L M, et al. Crystal structure and photoelectricity property of $\mathrm{Fe}(\mathrm{II} / \mathrm{III})$ coordination supermolecules. Chinese Sci Bull, 2010, 55: 124-130

24 Wang Y, Xue M, Xu J N, et al. Synthesis, crystal structure and properties of inorganic-organic hybrid polymers based on 8-hydroxylquinoline5-sulfonic acid. Sci China Ser B-Chem, 2009, 52: 1602-1608

25 Xie L H, Lin J B, Liu X M, et al. Organic ammonium ion-occluded flexible coordination polymers: Thermal activation, structure transformation and proton transfer. Sci China Ser B-Chem, 2010, 53: 2144-2151

26 Hou L, Zhang W X, Zhang J P, et al. An octacobalt cluster based, $(3,12)$-connected, magnetic, porous coordination polymer. Chem Commun, 2010, 46: 6311-6313

27 Guo Z G, Cao R, Wang X, et al. A multifunctional 3D ferroelectric and NLO-active porous metal-organic framework. J Am Chem Soc, 2009, 131: 6894-6895

28 Wang Z M, Hu K L, Gao S, et al. Formate-based magnetic metal-organic frameworks templated by protonated amines. Adv Mater, 2010, 22: 1526-1533

Open Access This article is distributed under the terms of the Creative Commons Attribution License which permits any use, distribution, and reproduction in any medium, provided the original author(s) and source are credited. 